\title{
Prevalence of Pediatric Functional Gastrointestinal Disorders Utilizing the Rome IV Criteria
}

\author{
Samantha G. Robin, BS ${ }^{1}$, Catherine Keller, BS ${ }^{1, *}$, Russell Zwiener, $\mathrm{MD}^{2}$, Paul E. Hyman, MD², Samuel Nurko, MD, MPH ${ }^{3}$,

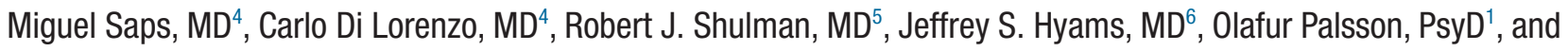 \\ Miranda A. L. van Tilburg, PhD ${ }^{1,7,8}$
}

Objective To assess the prevalence of functional gastrointestinal (GI) disorders in children 0-18 years old according to the newly established Rome IV diagnostic criteria as reported by parents in a representative community sample.

Study design A cross-sectional study in which mothers $(n=1255)$ of children aged $0-18$ years old in the US were recruited to complete an online survey about their child's Gl symptoms, quality of life (QoL), and other health conditions.

Results Based on the Rome IV criteria, $24.7 \%$ of infants and toddlers aged $0-3$ years and $25.0 \%$ of children and adolescents aged 4-18 years fulfilled symptom-based criteria for a functional Gl disorder. The most common functional Gl disorders were infant regurgitation among infants $(24.1 \%)$ and functional constipation among both toddlers $(18.5 \%)$ and children and adolescents $(14.1 \%)$. QoL was diminished in pediatric patients with functional GI disorders (median $=71.69$ vs median $=87.60 ; z=-11.41 ; P<.001$ ). Children were more likely to qualify for a functional GI disorder if their parent qualified for a functional Gl disorder (35.4\% vs $23.0 \%$; $P<.001)$.

Conclusions Based on Rome IV criteria, functional GI disorders are common in pediatric populations of all ages and are associated with decreased QoL. (J Pediatr 2018;195:134-9).

unctional gastrointestinal (GI) disorders are common in children of all ages worldwide. ${ }^{1-5}$ Functional GI disorders are currently best understood as bio-psychosocial disorders lacking an identifiable organic etiology, but affecting the complex interactions between the brain and gut. ${ }^{6}$ Different diagnostic criteria are established to diagnose infant and toddler functional GI disorders (0-3 years old) and child/adolescent functional GI disorders (4 years and older). Prevalence rates of pediatric functional GI disorders have been reported to be between $9.9 \%$ and $27.5 \%$ in children/adolescents ${ }^{7-11}$ and between $27 \%$ and $40.5 \%$ in infants and toddlers. ${ }^{1,5}$

In 2016, the Rome IV criteria for functional GI disorders were published, replacing the Rome III criteria established in 2006. ${ }^{6,12-14}$ The goal of the Rome IV process was to update the diagnostic tools based on new findings in the literature including new information on gut-brain interactions and microenvironments. ${ }^{6}$ These new criteria include 2 new diagnoses for children ages 4 years and older (functional nausea and functional vomiting), and several modifications to diagnostic criteria for existing diagnoses. The goal of this study was to evaluate the prevalence of GI symptoms suggestive of functional GI disorders among infants and toddlers ages $0-3$ years old and children and adolescents ages 4-18 years old according to the new Rome IV criteria.

\section{Methods}

Mothers of children ages 0-18 years old were recruited through online survey panels by CINT USA, Inc (Atlanta, GA; www.cint.com). CINT has 19 million registered panel members worldwide, consisting of individuals who have joined to answer a variety of surveys. CINT targeted recruitment among their panel members to mothers living in the US with predetermined quota-based sampling to ensure adequate representation of sex, race, and age groups. Mothers rather than fathers

$\begin{array}{ll}\text { GI } & \text { Gastrointestinal } \\ \text { IBD } & \text { Inflammatory bowel disease } \\ \text { IBS } & \text { Irritable bowel syndrome } \\ \text { QoL } & \text { Quality of life } \\ \text { QPGS-RIII } & \text { Questionnaire on Pediatric Gastrointestinal Symptoms-Rome III } \\ \text { RIV-PDQ } & \text { Rome IV Pediatric Diagnostic Questionnaire }\end{array}$

From the ${ }^{1}$ Department of Medicine, Division of Gastroenterology and Hepatology, Center for Functional Gl and Motility Disorders, University of North Carolina, Chapel Hill, NC; '2Department of Pediatrics, Louisiana State University, New Orleans, LA; ${ }^{3}$ Center for Motility and Functional Gastrointestinal Disorders, Boston Children's Hospital, Boston, MA; ${ }^{4}$ Division of Gastroenterology and Nutrition, Nationwide Children's Hospital, Columbus, OH; ${ }^{5}$ Children's Nutrition Research Center, Baylor College of Medicine Department of Pediatrics, Texas Children's Hospital, Houston, TX: ${ }^{6}$ Division of Digestive Diseases,Hepatology, and Nutrition, Connecticut Children's Medical Center, Hartford, CT;

${ }^{7}$ College of Pharmacy and Health Sciences, Campbell University, Buies Creek, NC; and ${ }^{8}$ School of Social Work University of Washington, Seattle, WA

${ }^{*}$ Contributed equally.

Sponsored by the Rome Foundation. P.H., S.N., M.S., C.D.L., R.S., J.H., O.P., and M.v.T. are part of the Rome IV Committee. The other authors declare no conflicts of interest.

Portions of this study were presented at the World Congress of Pediatric Gastroenterology, Hepatology and Nutrition, October 5-8, 2016, Montreal, Quebec and at Digestive Disease Week, May 6-9, 2017, Chicago, Illinois. 
were recruited to complete the survey because mothers are more likely to be the primary caregiver of their child and accompany their child to doctor's visits. Parent-child GI symptom overlap has also been more extensively studied previously in mothers. ${ }^{1}$

Data collection was completed during the first week of May 2016. Mothers were recruited to complete a survey on "child health," which was not identified as a survey relating to GI symptoms to prevent selection bias. If parents had more than 1 child age 0-18 years, they were asked to answer all questions about the child whose name was first in alphabetical order. Parents completed an online consent form and were then directed to complete a secure online survey using Qualtrics software (https://www.qualtrics.com/research-suite/). No identifying information was collected from responders or their children. This study was approved by the Institutional Review Board of the University of North Carolina (15-0647).

Mothers in this study completed the Rome IV Pediatric Diagnostic Questionnaire (RIV-PDQ) about their child's GI symptoms. The RIV-PDQ was developed by Rome IV committee members and is based on the Rome III questionnaire, the Questionnaire on Pediatric Gastrointestinal Symptoms-Rome III (QPGS-RIII), which has been validated in both children/ adolescents as well as infants and toddlers. ${ }^{15-18}$

Mothers also completed a questionnaire about their own GI symptoms, using the Functional Bowel Module of the Rome IV Diagnostic Questionnaire for Adults (an updated version of the Rome III questionnaire). ${ }^{19}$ This questionnaire has high specificity for irritable bowel syndrome (IBS), functional dyspepsia, and functional constipation and moderate sensitivity. ${ }^{19}$ To limit participant burden, only questions related to the most common functional GI disorders, IBS, functional dyspepsia, functional constipation, and functional diarrhea were included. All questionnaires can be requested from the Rome foundation (http://theromefoundation.org/).

Quality of life (QoL) was assessed using the PedsQL4.0 Generic Core Scale, which includes subscales assessing physical, emotional, social, and school/day care functioning..$^{20}$ Items were transformed from a scale of $0-5$ to $0-100$, with higher scores indicating higher QoL. Total QoL scores were averaged from the subscale scores with each subscale weighted evenly. If parents rated school/day care functioning questions not applicable for their child, summary scores were averaged excluding the school/day care functioning subscale. Separate parent-proxy scales were used for toddlers (ages 2-4 years), young children (ages 5-7 years), older children (ages 8-12 years), and teens (ages 13-18 years). QoL data were not collected for children less than 2 years old because this scale is only validated for children 2 years and older.

Demographic questions included parent and child age, sex, parent and child race/ethnicity, marital and cohabitation status, household income, parental education, proportion of time parent lives with child, and state of residence. Health questions included number of doctor's visits, school/ work absences, and hospital stays in the previous 6 months as well as use of common medications and common health problems.

\section{Statistical Analyses}

Averages and SDs or percentages of the sample were calculated as appropriate. The $t$ tests provided results for continuous variables and $\chi^{2}$ tests were used to analyze categorical variables. There were no missing data because the Qualtrics software required responses to all questions before continuing through the survey.

Several control measures were used to ensure quality of data. Responses were restricted to 1 entry per computer device, and responses were excluded if 3 repeated questions about the mother's own symptoms were answered inconsistently. Subjects were excluded from analyses if either the mother or child reported a personal history of cancer, celiac disease, or inflammatory bowel disease (IBD), or if the mother completing the survey lived with her child less than one-half of the time.

\section{Results}

A total of 1515 mothers completed the questionnaire, 368 of whom provided information about children $0-3$ years old and 1147 for children 4-18 years old. Data was excluded from respondents who had children with a history of celiac disease $(\mathrm{n}=14), \operatorname{IBD}(\mathrm{n}=7)$, or cancer $(\mathrm{n}=2)$, and mothers with a history of celiac disease $(n=17)$, IBD $(n=17)$, or cancer $(n=22)$, or who lived with their children less than one-half of the time $(n=3)$. Respondents were also excluded for inconsistent answers $(\mathrm{n}=195)$.

After exclusions, a total of 296 infants and toddlers and 959 children 4 years and older were included in the analyses. Table I provides demographic information on the included subjects. The sample shows a good distribution across races/ethnicity, race, age, and socioeconomic class as obtained per quotabased sampling. Mothers living in all states across the US were included.

\section{Infant/ Toddler Rome IV Diagnoses}

Table II provides the prevalence of functional GI disorders among infants and toddlers in the sample according to Rome IV criteria. Over one-third (37.9\%) of infants less than 1 year old met the diagnostic criteria for at least 1 functional GI disorder. Among toddlers, $21.4 \%$ met the criteria for at least 1 functional GI disorder. Among infants and toddlers combined, $24.7 \%$ qualified for at least 1 functional GI disorder. Among infants and toddlers meeting criteria for any functional GI disorder, 9.6\% met criteria for more than 1 functional GI disorder. Infant regurgitation was the most common disorder in infants, and functional constipation was the most common among toddlers (Table II). There were no infants with dyschezia and no infants or toddlers with functional diarrhea.

There were no significant differences between boys and girls in the number or type of functional GI disorders. Notably, no male infants met the criteria for infant colic, and 3 female infants fulfilled criteria $(P=.082)$. Race differences in prevalence and type of functional GI disorders were not calculated because most racial categories had less than 5 infants/toddlers meeting criteria for a functional GI disorder. 


\begin{tabular}{l}
\hline Demographics \\
\hline Sex (female) \\
Age \\
\\
Race/ethnicity \\
Hispanic \\
Caucasian \\
African American \\
Asian or Pacific Islander \\
Native American \\
Mixed or nondisclosed \\
Living arrangement \\
Household income \\
$<\$ 25000$ \\
$\$ 25000-\$ 50000$ \\
$\$ 50000-\$ 100000$ \\
$>\$ 100000$ \\
Parental education \\
Some high school \\
High school graduate or some college \\
College graduate or some graduate school (Associate or Bachelor degree) \\
Postgraduate degree \\
Q0L (ages $2-18$ y)
\end{tabular}

$N / A$, not applicable.

\section{Child/ Adolescent Rome IV Diagnoses}

Table III provides the prevalence of functional GI disorders among children and adolescents ages 4 years and older in the sample according to Rome IV criteria. Among children and adolescents ages 4 years and older, 25.0\% qualified for at least 1 functional GI disorder by Rome IV criteria. Children and adolescents in the study qualified for 11 of 12 functional GI disorders diagnosable in this age group; no subjects qualified for rumination. The most common functional GI disorders were functional constipation $(14.1 \%)$ and functional dyspepsiapostprandial distress syndrome (7.2\%).

Functional abdominal pain-not otherwise specified was more prevalent in female $(4.2 \%)$ than male subjects $(1.8 \%, P=.04)$.

Table II. Functional GI disorder prevalence in infants and toddlers according to Rome III and Rome IV criteria

\begin{tabular}{lcr}
\hline Diagnoses & Rome IV, N (\%) & Rome III* \\
\hline Infants (N = 58) & & \\
$\quad$ Infant regurgitation & $14(24.10 \%)$ & $25.90 \%$ \\
Infant colic & $3(5.20 \%)$ & $5.90 \%$ \\
Infant dyschezia & $0(0 \%)$ & $2.40 \%$ \\
Cyclic vomiting syndrome & $1(1.70 \%)$ & $0.00 \%$ \\
Rumination & $1(1.70 \%)$ & $2.40 \%$ \\
Functional constipation & $7(12.10 \%)$ & $4.70 \%$ \\
Functional diarrhea & $0(0 \%)$ & $2.40 \%$ \\
At Least 1 functional Gl disorder (infants) & $22(37.90 \%)$ & $27.10 \%$ \\
Toddlers (N = 238) & $5(2.10 \%)$ & \\
Cyclic vomiting syndrome & $5(2.10 \%)$ & $3.40 \%$ \\
Rumination & $44(18.50 \%)$ & $1.90 \%$ \\
Functional constipation & $0(0.00 \%)$ & $6.40 \%$ \\
Functional diarrhea & $51(21.40 \%)$ & Not reported
\end{tabular}

*Rome III prevalence data are from a previous study that used the same methods as the current study.

Functional dyspepsia-epigastric pain syndrome was more prevalent in male $(0.9 \%)$ than female subjects $(0.0 \%, P=.04)$. Aerophagia was more common in Hispanic children (5.5\%) than non-Hispanic children $(2.1 \%, P=.04)$. No other differences were found in prevalence by sex, race, or ethnicity.

Table III. Functional GI disorder prevalence in children greater than 4 years old according to Rome III and Rome IV criteria

\begin{tabular}{lcc}
\hline Diagnoses & Rome IV, N (\%) & Rome III $^{\star}$ \\
\hline Functional constipation & $135(14.10 \%)$ & $122(12.90 \%)$ \\
$\begin{array}{l}\text { Functional dyspepsia - postprandial } \\
\quad \text { distress syndrome }\end{array}$ & $69(7.20 \%)$ & - \\
$\begin{array}{l}\text { Functional dyspepsia - epigastric pain }_{\quad} \\
\quad \text { syndrome }\end{array}$ & $4(0.40 \%)$ & - \\
Functional dyspepsia - unspecified ${ }^{\dagger}$ & $\mathrm{N} / \mathrm{A}$ & $2(0.20 \%)$ \\
IBS & $49(5.10 \%)$ & $27(2.80 \%)$ \\
FAP NOS & $30(3.1 \%)$ & FAP $2(0.3 \%)$ \\
& & FAPS $8(0.8 \%)$ \\
Aerophagia & $25(2.60 \%)$ & $41(4.30 \%)$ \\
Cyclic vomiting syndrome & $19(2.00 \%)$ & $10(1.10 \%)$ \\
Functional vomiting & $13(1.40 \%)$ & - \\
Abdominal migraine & $11(1.10 \%)$ & $87(9.20 \%)$ \\
Functional nausea & $5(0.50 \%)$ & - \\
Nonretentive fecal incontinence & $2(0.20 \%)$ & $17(1.80 \%)$ \\
Rumination & $0(0 \%)$ & $0.00 \%$ \\
Any functional Gl disorder & $25.00 \%$ & $23.10 \%$
\end{tabular}

FAP, functional abdominal pain; FAPS, functional abdominal pain syndrome; NOS, not otherwise specified.

${ }^{*}$ Rome III prevalence data are from a previous study that used the same methods as the current study. ${ }^{2}$

†The 2 subcategories of functional dyspepsia (functional dyspepsia-postprandial distress syndrome and functional dyspepsia-epigastric pain syndrome) are new diagnostic categories unde Rome IV criteria. As such, the categories for functional dyspepsia do not match between Rome III and Rome IV data. 


\section{QoL and Medical Visits}

QoL data were available for 1129 children between the ages of 2 and 18 years old. The Shapiro-Wilkes test demonstrated that the QoL data were not normally distributed $(P<.001)$ and the Q-Q plot also showed deviations from normality (peaked data, kurtosis = 2.04). A Mann-Whitney test was used for group comparison for this reason, and it showed that children with a functional GI disorder had lower QoL scores (median $=71.69$, range $=0.0-100.0)$ than toddlers without a functional GI disorder (median $=87.60$, range $=0.0-100.0)$, by Mann-Whitney test $(\mathrm{z}=-11.41, P<.001)$.

Data on medical visits, hospital stays, and school and day care absences were not normally distributed by Q-Q plot (with kurtosis ranging from 28.3 to 70.4 and skewness from 4.6 to 7.4) and, therefore, were analyzed using the Mann-Whitney test. School and day care absences were significantly higher in children meeting criteria for a functional GI disorder (mean $=4.09$; mean rank $=696.51)$ vs those not meeting criteria for a functional GI disorder (mean $=2.38$; mean rank $=604.15 ; \mathrm{z}=-4.05 ; P<.001$ ). Children with functional GI disorders had significantly more medical visits in the past 6 months because of GI problems (mean $=0.92$; mean rank $=786.28$ ) compared with children not meeting criteria for a functional GI disorder (mean $=0.19$; mean rank $=573.28$; $\mathrm{Z}=-12.89, P<.001)$. Hospital stays in the past 6 months were also higher in children with functional GI disorders (mean $=0.49$; mean rank $=700.63$ ) compared with those not meeting functional GI disorder criteria (mean $=0.08$; mean rank $=602.76, \mathrm{Z}=-8.25, P<.001$ ). Mothers of children with functional GI disorders missed significantly more work because of their child's illness (mean $=1.66$; mean rank $=716.88$ ) than mothers of children without functional GI disorders $($ mean $=0.75 ;$ mean rank $=596.69 ; \mathrm{Z}=-6.68, P<.001)$.

\section{Parent-Child Overlap}

Among the mothers in the study, $18.2 \%$ qualified for a functional GI disorder with functional dyspepsia unspecified $(10.9 \%)$ and functional dyspepsia postprandial distress syndrome (8.4\%) being the most common. $6.8 \%$ of mothers met criteria for functional constipation, $4.8 \%$ for IBS, but none for functional diarrhea. The prevalence of functional GI disorders in children was significantly higher in children whose mothers also met criteria for a functional GI disorder compared with children of mothers who did not meet criteria (35.4\% vs $23.0 \%$; $P<.001$ ).

When analyzed by age group, infants and toddlers of mothers with a functional GI disorder were not significantly more likely to have a functional GI disorder than infants and toddlers of mothers who did not meet criteria ( $32.8 \%$ vs $22.4 \% ; P=.088$ ). Because functional GI disorders are a heterogeneous group of disorders, we examined if infants/toddlers and mothers overlapped in the same disorder. We were only able to do this for functional constipation, however, as it was the only disorder that was assessed in both mothers and infants/toddlers. Mothers with functional constipation were not more likely to have an infant or toddler who also qualified for Rome IV diagnostic criteria of functional constipation $(23.8 \%$ vs $16.7 \%$; $P=.407)$.
In contrast, children 4 years and older were more likely to qualify for a functional GI disorder if their mother also had a functional GI disorder $(35.2 \%$ vs $22.9 \% ; P<.001)$. For specific functional GI disorders, mothers with functional dyspepsia were more likely to have children who also met criteria for functional dyspepsia $(16.7 \%$ vs $6.5 \% ; P<.001)$, but mothers with functional constipation or IBS were not more likely to have children with these same diagnoses $(18.8 \%$ vs $13.7 \%$, $P=.27$ and $4.7 \%$ vs $5.1 \%, P=.89$ ). For this analysis, all subtypes of functional dyspepsia were grouped into 1 variable for mother and child.

\section{Discussion}

This study has established the prevalence of pediatric functional GI disorders according to the new Rome IV criteria. Prevalence was investigated in a racially diverse sample and included mothers and their children from all 50 states in the US and across a wide range of household incomes. The prevalence rates of pediatric functional GI disorders according to Rome IV criteria in this study can be compared with Rome III prevalence rates reported in 2 previous studies with nearly identical methods. ${ }^{1,2}$ Among infants and toddlers, overall prevalence of functional GI disorders using Rome IV criteria was found to be nearly one-quarter $(24.7 \%)$, which is comparable with the $27.1 \%$ prevalence of functional GI disorders in infants and toddlers reported using Rome III criteria. ${ }^{1}$ Among children and adolescents ages 4-18 years old, overall Rome IV prevalence rates $(25.0 \%)$ similarly were not changed meaningfully from previously reported Rome III prevalence rates $(23.1 \%){ }^{2}$

The purpose of Rome IV was to update clinical and research definitions of functional GI disorders to reflect new insights and provide more precise diagnostic criteria for clinicians and researchers. ${ }^{14}$ In this study, the prevalence of functional constipation in infants and toddlers increased from $4.7 \%$ of infants and $9.4 \%$ of toddlers to $17.2 \%$ of infants and toddlers combined. Changes to the functional constipation Rome criteria did not seem responsible for this increase in prevalence in functional constipation. Rather, parents in this current study endorsed a "history of large-diameter stools that may obstruct the toilet after the acquisition of toileting skills" more frequently than parents in the Rome III study ${ }^{1}$ with similar methodology ( $12.8 \%$ vs $4.2 \%$ ), and this difference seemed to best account for the increase in prevalence.

No infants or toddlers met criteria for functional diarrhea in our study sample and Rome III found a prevalence of $2.40 \%$ of infants and $6.40 \%$ of toddlers meting criteria for functional diarrhea. Rome IV criteria eliminated defecation during sleep as a criterion because defecation during sleep is relatively common in young children. ${ }^{14}$ Eliminating nonspecific and common symptoms may explain the decrease in prevalence of functional diarrhea under the Rome IV criteria.

Among children and adolescents ages 4 years and older, the prevalence of abdominal migraine decreased substantially from Rome III (9.2\%) to Rome IV (1.1\%). This was the intention of the Rome committee, given that Rome III rates of abdomi- 
nal migraine were likely overestimated under Rome III because of criteria nonspecificity, ${ }^{15,21}$ so both diagnostic criteria and questionnaire items were revised to address this concern. ${ }^{6}$ The Rome IV prevalence data are more reflective of pre-Rome III studies and likely reflect a more realistic prevalence figure for this disorder. ${ }^{22,23}$

Compared with our Rome III prevalence study, it appears that the prevalence of IBS almost doubled from Rome III (2.8\%) to Rome IV (5.1\%). However, IBS was low in the Rome III prevalence study compared with other studies (4.9\%-7.0\%), $)^{22-24}$ and this increase may just reflect a return to normal values.

The diagnosis of infant dyschezia was relatively rare, but the prevalence was different in this study vs Rome III. No infants met the Rome IV criteria for infant dyschezia, despite $2.4 \%$ of infants meeting the Rome III criteria in a previous study. ${ }^{1}$ Rates for nonretentive fecal incontinence, decreased from $1.8 \%$ in Rome $\mathrm{III}^{2}$ to $0.2 \%$ in Rome IV. These small changes in uncommon diagnoses most likely do not reflect an actual change in prevalence because of changes in diagnostic criteria.

Several new diagnostic entities were added and are in need of special mention. Functional vomiting and functional nausea were new diagnoses in Rome IV. Despite the low prevalence $(\sim 1 \%)$ of these diagnoses in this sample, they may still represent important diagnostic categories in the pediatric population. In addition, 2 subtypes of functional dyspepsia were included in Rome IV. The first subtype, postprandial distress syndrome, does not require pain to be present (which was required in Rome III), and it was the most common subtype, characterizing $94.5 \%$ of patients with functional dyspepsia. The rate of subjects reporting pain with functional dyspepsia (now the epigastric pain syndrome subtype of functional dyspepsia) was similar to previously reported prevalence of functional dyspepsia. ${ }^{2}$

Children with functional GI disorders demonstrated lower QoL compared with those not meeting criteria, underscoring the clinical relevance of these diagnoses. These findings are concordant with previous findings of decreased QoL associated with pediatric functional GI disorders. ${ }^{2,25,26}$ Increased doctor and hospital visits as well as school, work, and daycare absences further demonstrates the healthcare and productivity costs of these disorders.

Previous studies have identified overlap between parent and child functional GI symptoms. ${ }^{1}$ This study seems to suggest that the association between mother and child functional GI disorder diagnoses is likely stronger in older children, perhaps because the nature of functional GI disorders in older children is more similar to adult functional GI disorders, as opposed to infant and toddler diagnoses which are significantly limited by the child's ability to recognize, report, and describe symptoms. These associations between parent and child functional GI disorder diagnoses are likely because of a combination of both genetic and environmental factors including but not limited to parental response to GI symptoms, diet, and healthcare seeking behavior. ${ }^{27}$ In addition, this study only examined the relationship between mother and child functional GI disorder diagnoses, but did not consider the medical history of the subjects' fathers or healthcare seeking behaviors of other caretakers. Further studies are needed to understand the overlap between GI symptoms in infants/toddlers and their parents.

Despite the strengths of this study in evaluating pediatric GI prevalence in a large and diverse population, the study also has several limitations. First, functional GI disorder prevalence data were collected in this study using the RIV-PDQ, which has been validated in adults, but not yet in those below the age of 18 years. However, given this questionnaire's similarity to the validated QPGS-RIII, it is likely that the RIVPDQ performs similarly to the QPGS-RIII. ${ }^{15}$

Another limitation is that subjects in this study were recruited through online panel surveys, and this strategy has both benefits and risks. The greatest benefit of this method is that predetermined quotas could be used for obtaining a diverse sample, including demographics typically underrepresented in community surveys, making the findings more generalizable to the US pediatric population. Surveys were completed anonymously, and subjects had no interaction with a researcher, minimizing parent response bias. The description of this survey did not indicate the survey was about child GI symptoms, minimizing selection bias of the parents choosing to complete this survey. Despite these controls, evidence exists that selfreported data obtained from online panels may be different than responses collected through other means, ${ }^{1,28}$ notably that online survey responders may be more willing to report socially undesirable behavior. It is unclear whether online survey respondents differ from research samples responding in person (sample bias), or whether responses are influenced by the survey format. Online surveys may also be easier to answer indiscriminately or fraudulently. However, the data quality strategies employed, such as requiring respondents to register and confirm identity, only allowing 1 response per IP address, and excluding surveys with inconsistent responses to repeated questions all improve data quality and decrease fraudulent or indiscriminate data survey responses.

Another limitation of this study is that the community survey format cannot eliminate the possibility of organic medical causes for reported GI symptoms. However, previous research demonstrates that it is unlikely to find a medical cause of most symptoms that fit diagnostic criteria of functional GI disorders. ${ }^{29,30}$

Lastly, this study used parent reporting of child symptoms. Agreement between parent and child on the QPGSRome III was low for certain disorders. ${ }^{17,31}$ Especially in older children, parents may not be aware of nonobservable, undisclosed symptoms. This effect may explain why no subjects in this study were found with rumination because the symptoms of this disorder could occur without parental knowledge. ${ }^{2,8}$ Furthermore, parents' scoring of QoL for their children with functional abdominal pain has been shown to be lower than children's self-reported scores. ${ }^{32}$ Therefore, future studies should include child-reported QoL data in appropriate age groups.

In conclusion, functional GI disorders according to the Rome IV criteria are common among children. Prevalence figures are comparable for most conditions between the Rome III and Rome IV criteria although some significant changes were found such as in the prevalence rates of abdominal migraine. In 
addition, this study reports on prevalence figures of new diagnostic criteria such as functional nausea and vomiting. Longitudinal studies are needed to assess diagnostic stability and the impact of functional GI disorders throughout childhood and into adulthood.

Submitted for publication Sep 5, 2017; last revision received Nov 7, 2017; accepted Dec 4, 2017

Reprint requests: Miranda A. L. van Tilburg, PhD, College of Pharmacy and Health Sciences, Campbell University, 180 Main St, PO Box 1090, Buies

Creek, NC 27506. E-mail: vantilburg@ campbell.edu

\section{References}

1. van Tilburg MAL, Hyman PE, Walker L, Rouster A, Palsson OS, Kim SM, et al. Prevalence of functional gastrointestinal disorders in infants and toddlers. J Pediatr 2015;166:684-9.

2. Lewis ML, Palsson OS, Whitehead WE, van Tilburg MAL. Prevalence of functional gastrointestinal disorders in children and adolescents. J Pediatr 2016;177:39-43, e3.

3. van den Berg MM, Benninga MA, Di Lorenzo C. Epidemiology of childhood constipation: a systematic review. Am J Gastroenterol 2006;101:24019.

4. Vandenplas Y, Abkari A, Bellaiche M, Benninga M, Chouraqui JP, Çokura F, et al. Prevalence and health outcomes of functional gastrointestinal symptoms in infants from birth to 12 months of age. J Pediatr Gastroenterol Nutr 2015;61:531-7.

5. Chogle A, Velasco-Benitez CA, Koppen IJ, Moreno JE, Ramírez Hernández CR, Saps M. A population-based study on the epidemiology of functional gastrointestinal disorders in young children. J Pediatr 2016;179:13943, e1.

6. Drossman DA. Functional gastrointestinal disorders: history, pathophysiology, clinical features and Rome IV. Gastroenterology 2016.

7. Dhroove G, Saps M, Garcia-Bueno C, Leyva Jiménez A, RodriguezReynosa LL, Velasco-Benítez CA. Prevalence of functional gastrointestinal disorders in Mexican schoolchildren. Rev Gastroenterol Mex 2017;82: 13-8.

8. Bhatia V, Deswal S, Seth S, Kapoor A, Sibal A, Gopalan S. Prevalence of functional gastrointestinal disorders among adolescents in Delhi based on Rome III criteria: a school-based survey. Indian J Gastroenterol 2016;35:294-8.

9. Udoh E, Devanarayana NM, Rajindrajith S, Meremikwu M, Benninga MA. Abdominal pain-predominant functional gastrointestinal disorders in adolescent Nigerians. J Pediatr Gastroenterol Nutr 2016;62:588-93.

10. Bouzios I, Chouliaras G, Chrousos GP, Roma E, Gemou-Engesaeth V. Functional gastrointestinal disorders in Greek Children based on ROME III criteria: identifying the child at risk. Neurogastroenterol Motil 2017;29:18.

11. Játiva E, Velasco-Benítez CA, Koppen IJN, Játiva-Cabezas Z, Saps M. Prevalence of functional gastrointestinal disorders in schoolchildren in Ecuador. J Pediatr Gastroenterol Nutr 2016;63:25-8.

12. Drossman DA, Dumitrascu DL. Rome III: new standard for functional gastrointestinal disorders. J Gastrointestin Liver Dis 2006;15:237-41.

13. Drossman DA. Rome III: the new criteria. Chin J Dig Dis 2006;7:181-5.

14. Benninga MA, Faure C, Hyman PE, St James Roberts I, Schechter NL, Nurko S. Childhood functional gastrointestinal disorders: neonate/ toddler. Gastroenterology 2016.

15. van Tilburg MAL, Rouster A, Silver D, Pellegrini G, Gao J, Hyman PE. Development and validation of a Rome III functional gastrointestinal disorders questionnaire for infants and toddlers. J Pediatr Gastroenterol Nutr 2016;62:384-6.

16. Baber KF, Anderson J, Puzanovova M, Walker LS. Rome II versus Rome III classification of functional gastrointestinal disorders in pediatric chronic abdominal pain. J Pediatr Gastroenterol Nutr 2008;47:299-302.

17. van Tilburg MAL, Squires M, Blois-Martin N, Leiby A, Langseder A. Test of the child/adolescent Rome III criteria: agreement with physician diagnosis and daily symptoms. Neurogastroenterol Motil 2013;25:302e246.

18. Saps M, Nichols-Vinueza DX, Mintjens S, Pusatcioglu CK, VelascoBenítez CA. Construct validity of the pediatric Rome III criteria. J Pediatr Gastroenterol Nutr 2014;59:577-81.

19. Palsson OS, Whitehead WE, van Tilburg MAL, Chang L, Chey W, Crowell MD, et al. Development and Validation of the Rome IV diagnostic Questionnaire for Adults. Gastroenterology 2016;150:1481-91.

20. Varni JW, Burwinkle TM, Seid M, Skarr D. The PedsQL 4.0 as a pediatric population health measure: feasibility, reliability, and validity. Ambul Pediatr 2003;3:329-41.

21. Hyams JS, Di Lorenzo C, Saps M, Shulman RJ, Staiano A, van Tilburg M. Functional disorders: children and adolescents. Gastroenterology 2016;150:1456-68.

22. Devanarayana NM, Adhikari C, Pannala W, Rajindrajith S. Prevalence of functional gastrointestinal diseases in a cohort of Sri Lankan adolescents: comparison between Rome II and Rome III criteria. J Trop Pediatr 2011;57:34-9.

23. Saps M, Nichols-Vinueza DX, Rosen JM, Velasco-Benítez CA. Prevalence of functional gastrointestinal disorders in Colombian school children. J Pediatr 2014;164:542-5, e1.

24. Devanarayana NM, Mettananda S, Liyanarachchi C, Nanayakkara N, Mendis N, Perera N, et al. Abdominal pain-predominant functional gastrointestinal diseases in children and adolescents: prevalence, symptomatology, and association with emotional stress. J Pediatr Gastroenterol Nutr 2011;53:659-65.

25. Varni JW, Bendo CB, Nurko S, Shulman RJ, Self MM, Franciosi JP, et al. Health-related quality of life in pediatric patients with functional and organic gastrointestinal diseases. J Pediatr 2015;166:85-90.

26. Koloski NA, Talley NJ, Boyce PM. Epidemiology and health care seeking in the functional GI disorders: a population-based study. Am J Gastroenterol 2002;97:2290-9.

27. Levy RL, Langer SL, Walker LS, Feld LD, Whitehead WE. Relationship between the decision to take a child to the clinic for abdominal pain and maternal psychological distress. Arch Pediatr Adolesc Med 2006;160:9615.

28. Link MW, Mokdad AH. Alternative modes for health surveillance surveys: an experiment with web, mail, and telephone. Epidemiology 2005;16:7014.

29. Dhroove G, Chogle A, Saps M. A million-dollar work-up for abdominal pain: is it worth it? J Pediatr Gastroenterol Nutr 2010;51:57983.

30. Di Lorenzo C, Colletti RB, Lehmann HP, Boyle JT, Gerson WT, Hyams JS, et al. Chronic abdominal pain in children: a technical report of the American academy of pediatrics and the North American society for pediatric gastroenterology, hepatology and nutrition. J Pediatr Gastroenterol Nutr 2005;40:249-61.

31. Czyzewski DI, Lane MM, Weidler EM, Williams AE, Swank PR, Shulman RJ. The interpretation of Rome III criteria and method of assessment affect the irritable bowel syndrome classification of children. Aliment Pharmacol Ther 2011;33:403-11.

32. Youssef NN, Murphy TG, Langseder AL, Rosh JR. Quality of life for children with functional abdominal pain: a comparison study of patients' and parents' perceptions. Pediatrics 2006;117:54-9. 